\title{
Eigenschaften von Samariumsulfidschichten für Sensoranwendungen
}

\author{
A. Delan ${ }^{1}$, R. Ngoumeni', K. Vondkar ${ }^{3}$, D. Glöß³, G. Gerlach ${ }^{1}$ \\ ${ }^{1}$ Technische Universität Dresden, Institut für Festkörperelektronik, Dresden, Deutschland \\ 2Sindlhauser Materials GmbH, Kempten, Deutschland \\ ${ }^{3}$ Fraunhofer-Institut für Organische Elektronik, Elektronenstrahl- und Plasmatechnik, Dresden, \\ Deutschland \\ annekatrin.delan@tu-dresden.de
}

\begin{abstract}
Zusammenfassung
Samariummonosulfid (SmS) ist ein Typ-n-Halbleiter mit einer Energielücke von etwa 0,2 eV. Es weist eine Reihe besonderer Eigenschaften hinsichtlich seiner Energiebandstruktur auf, aus der sich ein starker Einfluss mechanischer Effekte auf den Widerstand und ausgezeichnete optische Schalteigenschaften ergeben. Damit ist SmS für verschiedene Anwendungen in der Sensorik (z.B. Drucksensoren, Dehnungsmessstreifen) und in der Optik interessant. Dieser Beitrag stellt die Herstellung von SmS-Targets für die Abscheidung dünner SmS-Schichten mittels Magnetron-Sputtern vor. Die SmS-Schichten werden bzgl. der Stöchiometrie und der elektrischen und optischen Eigenschaften charakterisiert und Sensoranwendungen diskutiert.
\end{abstract}

Keywords: Samariumsulfid,Magnetron-Sputtern,SmS-Target,optische und elektrische Eigenschaften,EDX

\section{Einleitung}

Samariummonosulfid (SmS) ist ein Typ-nHalbleiter mit einer Energielücke von etwa $0,2 \mathrm{eV}$. Durch seine spezielle Energiebandstruktur hat es in den letzten Jahrzehnten einige Aufmerksamkeit in der Fachliteratur erregt. Der starke Einfluss verschiedener mechanischer Effekte auf den Widerstand von SmS und die ausgezeichnete optische Schalteigenschaft machen das Material für verschiedene Anwendungen im Sensorik- (z.B. Drucksensoren, Dehnungsmesstreifen) und im optischen Bereich attraktiv.

Dünnfilme aus SmS können durch eine Vielzahl von Verfahren hergestellt werden. Allerdings führte das reaktive Sputtern von $\mathrm{Sm}$ in $\mathrm{H}_{2} \mathrm{~S}$ zu pulverförmigen, nichtanhaftenden und chemisch instabilen Schichten [1]. In jüngerer Zeit bewiesen z.B. Volodin und Kaminskii das große Potenzial von SmS als Material für die Herstellung von Dehnungsmessstreifen und Drucksensoren für Raumfahrzeuganwendungen [2]. Miodushevsky et.al. optimierten den Schichtwiderstand von SmS durch Laserpulse [3]. Miodushevsky hatte auch mit der Herstellung von SmS-Targets experimentiert. Dabei hatte sich jedoch gezeigt, dass diese Oxide enthielten, weil während des Herstellungsprozesses kein ausreichendes Vakuum sichergestellt werden konnte.

In dieser Arbeit wurden SmS-Targets für die Abscheidung mit dem Magnetron-Sputterprozess entwickelt und hergestellt sowie SmSSchichten durch RF- und DC-MagnetronSputtern mit einem Magnetron mit 2" Targetdurchmesser abgeschieden. Die hergestellten SmS-Schichten wurden charakterisiert und die Schichtdicke- und Beschichtungsrateverteilung mittels Tastschnittmessung bestimmt. Weiterhin erfolgten Vierspitzenmessungen zur Bestimmung der Leitfähigkeit, IR- (FTIR) und UVIVISspektroskopische Untersuchungen zur optischen Charakterisierung und zum Nachweis von Sauerstoff- und Wasserstoff-Verunreinigungen sowie REM/EDX-Messungen zur Bestimmung der Stöchiometrie.

Die Untersuchung der Langzeitstabilität sowohl der SmS-Targets als auch der SmS-Schichten erfolgte mit dem Ziel, die praktische Anwendbarkeit abzuschätzen. 


\section{Target-Herstellung}

Die SmS-Targets wurden für die Abscheidung von Dünnschichten mittels Magnetron-Sputtern entwickelt. Zur Herstellung von Samariummonosulfid (SmS) ist das Verständnis der Eigenschaften der einzelnen Rohstoffe (Samarium und Schwefel) in Abhängigkeit von der Temperatur von fundamentaler Bedeutung. Deshalb wurden für die Targets die Ausgangsmaterialien, Zwischen- und Endprodukte (gepresste SmS-Targets) mittels thermischer Untersuchungen (DifferenzThermoanalyse (DTA) und Massenspekroskopie (MS)) von $30^{\circ} \mathrm{C}$ bis $1490^{\circ} \mathrm{C}$ in $5 \mathrm{~K}$-Schritten, mittels REM/EDX und zusätzlich mittels XRD charakterisiert.

Durch die thermischen Untersuchungen wurden in der DTA zwei kleine, endotherme Peaks bei ca. $110 \ldots 113^{\circ} \mathrm{C}$ und bei $280 \ldots$ $312{ }^{\circ} \mathrm{C}$ nachgewiesen, danach erfolgt ein starker exothermer Anstieg. Im Thermogram der TG wurde bei ca. $300{ }^{\circ} \mathrm{C}$ eine Massenabnahme von ca. $18 \%$ infolge der Oxidation von Schwefel beobachtet. Dabei bilden sich Schwefeloxide, die als $\mathrm{SO}$ und $\mathrm{SO}_{2}$ gasförmig freigesetzt werden.

Nicht gebundener Schwefel entweicht bei ca. 280 bzw. $312{ }^{\circ} \mathrm{C}$ als $\mathrm{SO}_{2}$ oder elementar. Die Massenzunahme von ca. 9,0 .. 9,5\% hat seine Ursache vermutlich in der Oxidation von Samarium zu $\mathrm{SmO}_{x}$ und durch die zusätzliche Phasenumwandlung in M-SmS (gelblich). Weiterhin wird durch die Auswertung der MS mittels QMID (Quadrupol-Massen-lonenDetektor) bei m:17 und m:18 ein großer Peak bei ca. $600{ }^{\circ} \mathrm{C}$ und ein kleiner Peak bei ca. $800^{\circ} \mathrm{C}$ sichtbar, dies bedeutet eine Wasserfreisetzung aus der Probe.

Die Bildung von SmS wurde mittels REM/EDX nachgewiesen (Abb. 1 und 2). Dabei bestätigte sich, dass in der bei $1000^{\circ} \mathrm{C}$ getemperten Probe teilweise mehr Schwefel als Samarium, aber in ähnlichen Mengen nachweisbar sind. Außerdem befindet sich meist etwas Sauerstoff an den Oberflächen. Nach Erhitzen auf $1000^{\circ} \mathrm{C}$ sind im XRD die typischen SmSPeaks sichtbar (Abb. 3). Weiterhin zeigten die Targets bei oberflächlicher Reibung einen kurzzeitigen Farbwechsel von schwarz zu gelb.

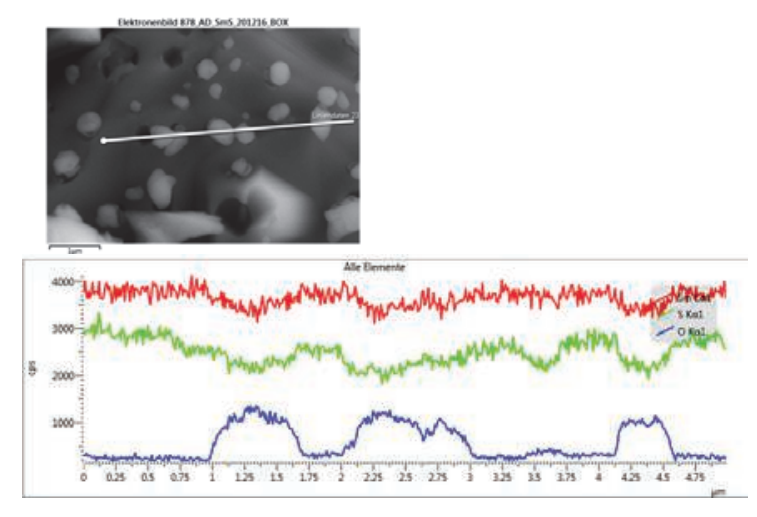

Abb. 1: REM und Linienspektrum der bei $1000^{\circ} \mathrm{C}$ getemperten SmS-Probe (rot Sm, grün $\mathrm{S}$, blau O), großes Partikel mit kleinen aufliegenden Partikeln

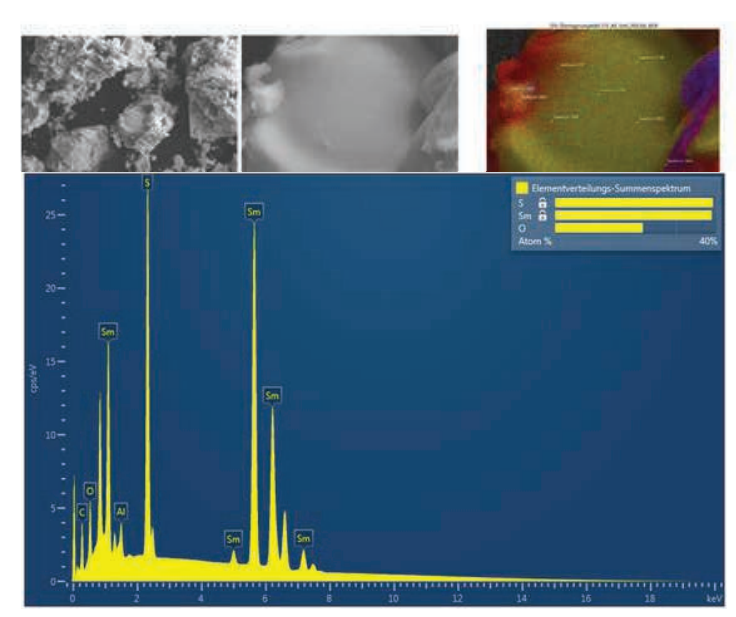

Abb. 2: REM, Überlagerungsbild und Elementverteilungs-Summenspektrum der bei $1000^{\circ} \mathrm{C}$ getemperten Probe (rot Sm, grün S, blau O), Partikel mit großer ebener Fläche

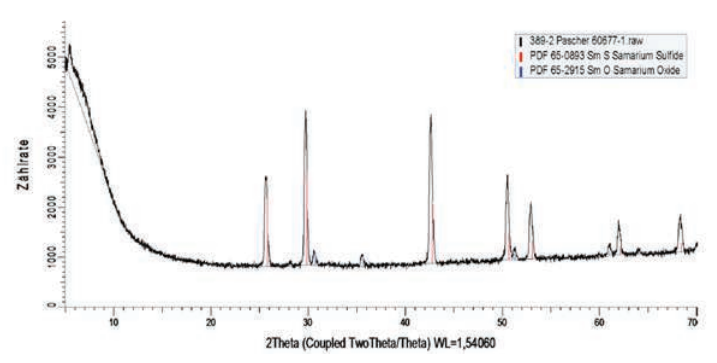

Abb. 3: $X R D$ an der SmS-Probe nach Erhitzen auf $1000{ }^{\circ} \mathrm{C}$

Die Rohstoffe Samarium und Schwefel wurden unter Inertgasbedingungen in Pulverform eingewogen und gemischt. Die Synthese von SmS findet in zwei Schritten statt. Der erste Schritt ist eine Vorstufenreaktion bei der Sm und $S$ ineinander diffundieren. Dabei soll 
Schwefel in die Flüssigphase übergehen und sich mit Samarium verbinden. In einem zweiten Schritt findet die eigentliche Kristallisation statt.

Das gemahlene SmS Pulver wurde in Formen eingefüllt und in einer CIP- (kalt-isostatisches Pressen) Anlage (Target 1, Abb. 3 links) oder uniaxial (Target 2, Abb.3 rechts) gepresst, um die 2"-SmS-Targets herzustellen. Die Targets 3 und 4 sind analog Target 1 kalt isostatisch gepresst.

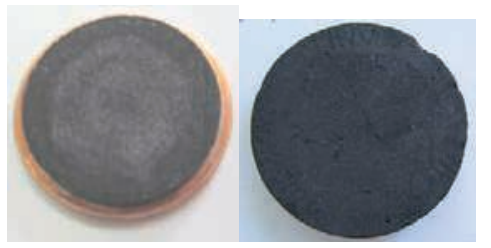

Abb. 3: SmS-Targets

\section{Präparation der Schichten}

Es wurden SmS-Schichten mit MagnetronSputtern hergestellt. Dies erfolgte an einer Beschichtungsanlage PLS 570 (Pfeiffer) mit einem 2"-Magnetron (Torus, Kurt J. Lesker Company, Abb. 4). Dabei wurde Objektträgerglas (OT-Glas, zwei oder ein Stück) und 4" SiWafer (einseitig und beidseitig poliert, <100>, p-leitend/Bor, 1/4 Si-Wafer mit einem Stück OTGlas) beschichtet. Tab. 1 zeigt die Beschichtungsbedingungen für die im Weiteren untersuchten Proben auf OT-Glas und Si.

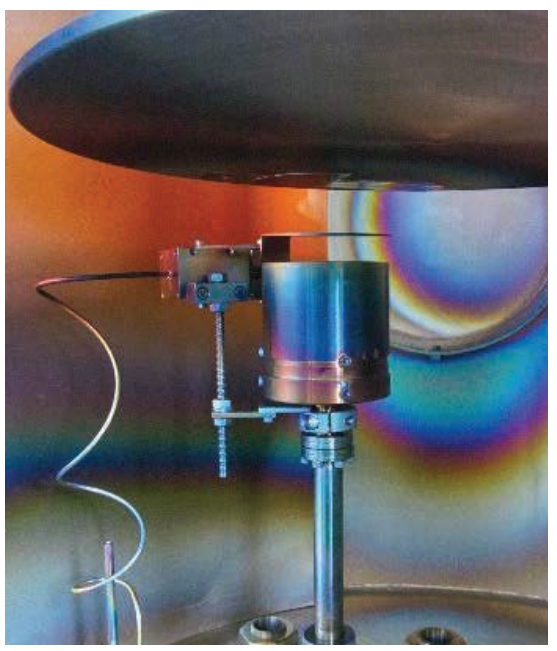

Abb. 4: 2"-Magnetron (Kurt J. Lesker Co.) in der Beschichtungsanlage PLS 570 (Pfeiffer)

\section{Charakterisierungsmethoden}

Die Untersuchung der Eigenschaften der SmSSchichten erfolgte
- mittels Tastschnittmessungen zur Ermittlung der Schichtdicken- und Beschichtungsrateverteilung,

- mit Vierspitzenmessungen zur Bestimmung der Leitfähigkeit,

- mit FTIR- und UVIVIS-Spektroskopie zur optischen Charakterisierung und zum Nachweis von Sauerstoff- und Wasserstoff-Verunreinigungen, sowie

- mit REM/EDX-Untersuchungen zur Ermittlung der Stöchiometrie.

Untersuchungen zu speziellen Anwendungseigenschaften, z.B. der Dehnungseigenschaften, sind geplant.

Tab. 1: Beschichtungsbedingungen der Proben

\begin{tabular}{|c|c|}
\hline Probe & Bedingungen \\
\hline 1 & RF, 50 W, 0.4 Pa, Target 1 \\
\hline 2 & RF, 75 W, 0.4 Pa, Target 1 \\
\hline 3 & RF, 50 W, 0.4 Pa, Target 1 \\
\hline 4 & $\mathrm{DC}, 25 \mathrm{~W}, 0.4 \mathrm{~Pa}$, Target 1 \\
\hline 5 & $\mathrm{RF}, 60 \mathrm{~W}, 0.4 \mathrm{~Pa}$, Target 1 \\
\hline 6 & $\mathrm{RF}, 60 \mathrm{~W}, 0.4 \mathrm{~Pa}$, Target 2 \\
\hline
\end{tabular}

\section{Eigenschaften der SmS-Schichten}

In Abb. 5 und 6 sind Fotos der Proben 3 und 4 zu sehen. Die Bereiche der Farben sind typisch. Es gibt meist drei Bereiche: die Mitte, gegenüber dem Sputtergraben und der Außenbereich. Die nicht beschichteten Bereiche wurden durch Aufkleben von Kaptonband erzeugt und dienen der Schichtdickenmessung an einer Stufe nach Entfernung des Kaptonbandes.

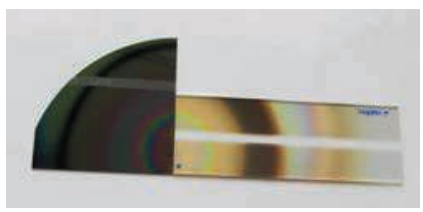

Abb. 5: Fotos der SmS-Probe 3 (links Si, rechts OT-Glas)

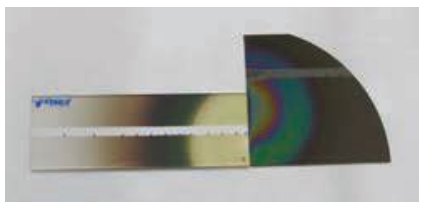

Abb. 6: Fotos der SmS-Probe 4 (links OTGlas, rechts Si)

Im Folgenden sind die StöchiometrieErgebnisse als Sm/S-Verhältnis, welches durch EDX-Untersuchungen auf den Targets ermittelt wurde, dargestellt:

- Target 1 im Sputtergraben: 0,77, 
- $\quad$ Target 2: 0,85,

- $\quad$ Target 3: 1,42,

- $\quad$ Target 4: 1,32.

Die ersten zwei Targets haben einen Schwefel Überschuss, das dritte und vierte Target einen Samarium Überschuss. Abbildung 7 zeigt die Stöchiometrie-Ergebnisse als Sm/S-Verhältnis, welches durch EDX-Untersuchungen auf den hergestellten SmS-Schichten auf Si-Substrat bestimmt wurde. Die Proben 1, 2 und 3 sind im Radiusbereich um 40 mm stöchiometrisch.

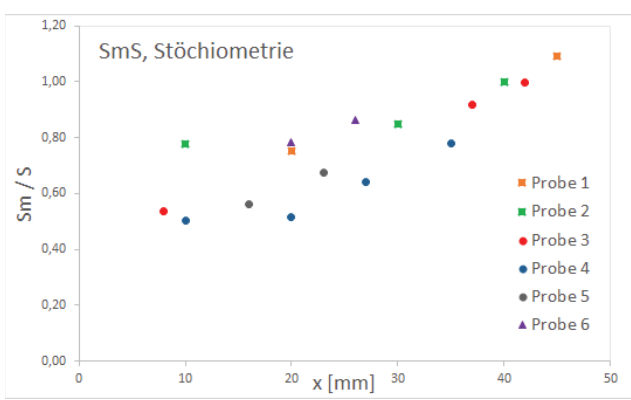

Abb. 7: Sm/S-Verhältnis der SmS-Schichten

In Abb. 8 sind die Ergebnisse der Tastschnittmessungen auf dem OT-Glas dargestellt. Die Beschichtungsrate war im Bereich gegenüber dem Sputtergraben und einem Radius einige $\mathrm{mm}$ darüber hinaus maximal. Bei kleineren Radien ist die Beschichtungsrate bei typischen Beschichtungsbedingungen vermindert.

Nach Ausbau des Targets 1 zur EDX Messung wurde eine dauerhafte Verminderung der maximalen Schichtdicke bei gleicher Beschichtungszeit festgestellt (Probe 1 und 2 erste Versuche vor Target Ausbau).

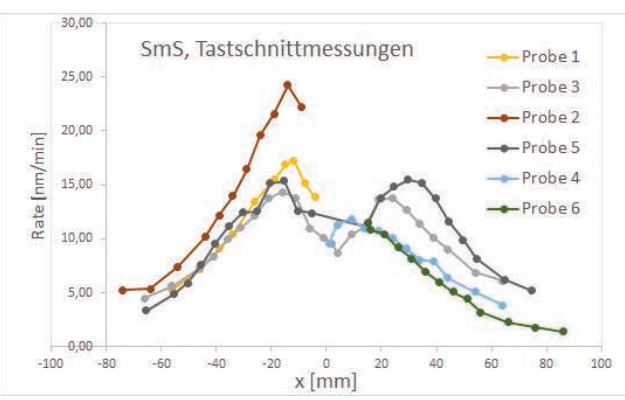

Abb. 8: Lokale Verteilung der Beschichtungsrate der SmS-Proben

Die Bestimmung der Leitfähigkeit erfolgte mittels der Vierspitzenmessung sowohl an den Targets als auch an den Proben. Folgende Werte für die Targets wurden ermittelt:

- $\quad$ Target 1: $110 \ldots 120 \Omega / \square$,

- $\quad$ Target 2: $130 \ldots 150 \Omega / \square$,

- $\quad$ Target 3: 0,3 $\Omega / \square$,

- $\quad$ Target 4: 0,21 $\Omega / \square$.

Die Leitfähigkeit der ersten beiden Targets ist mit der von einem ITO-Target (ca. $120 \Omega / \square$ ) vergleichbar. Die Targets 2 und 3 sind durch den Samarium Überschuss leitfähiger als die zwei anderen Targets mit Schwefel Überschuss.

Minimal traten folgende Leitfähigkeitswerte der Proben auf (außerhalb eines optimalen Bereiches sind die Proben isolierend):

- Probe 1 und 2: $255 \Omega / \square$,

- Probe 3: $241 \Omega / \square$,

- Probe 4: $248 \Omega / \square$,

- Probe 5: $21 \Omega / \square$,

- Probe 6: überall isolierend.

Die IR Messungen (Perkin Elmer S2000, Reflexionsmessung, Messbereich 390 bis $8000 \mathrm{~cm}^{-1}$ ) an den Probe 4 und 5 zeigen einen Peak bei ca. $1115 \mathrm{~cm}^{-1}$ bzw. $1100 \mathrm{~cm}^{-1}$, der nach [4] den Reflexion-Peaks von SmS zugeordnet werden kann (Abb. 9).

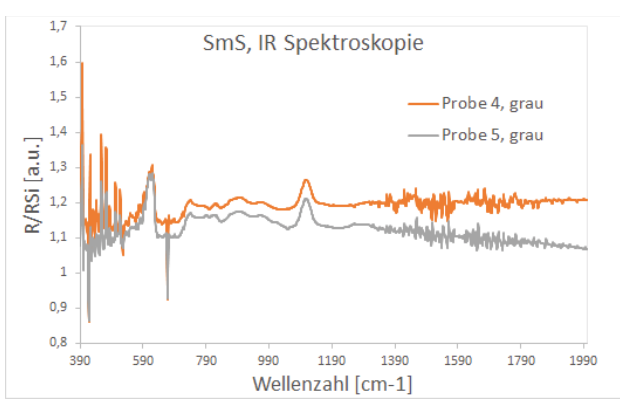

Abb. 9: Ausschnitt aus IR-Spektren an den SmS-Proben 5 und 6

Die UVIVIS-Messungen (Perkin Elmer Lambda 950) erfolgten im Wellenlängenbereich von 300 bis $2000 \mathrm{~nm}$ auf den SmS-Schichten auf OT-Glas.

In Abb. 10 ist ein Vergleich an drei Orten von Probe 3 zu sehen. Über dem Target und daneben ist die Probe bis 500 bzw. $750 \mathrm{~nm}$ absorptionsarm. Dies betrifft auch die Schicht, die im sichtbaren Bereich dunkel erscheint. Im Randbereich ist die Probe ab ca. $60 \mathrm{~mm}$ absorbierend.

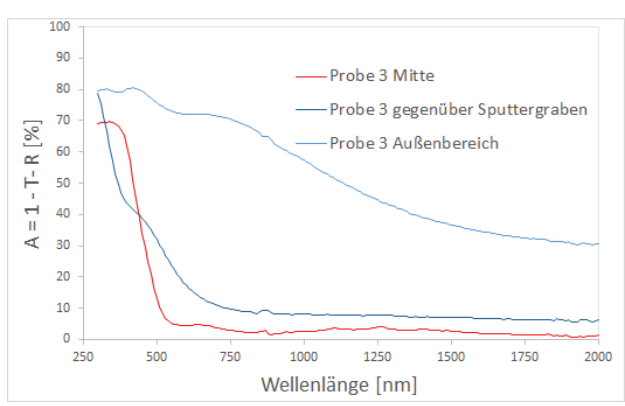

Abb. 10: Absorption ermittelt aus den UVIVISSpektren Probe 3 
In Abbildung 11 sind für den Bereich über dem Sputtergraben und daneben die Spektren der Proben dargestellt.

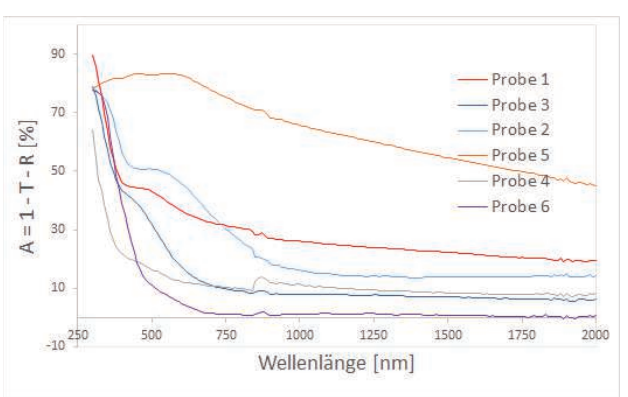

Abb. 11: Vergleich der Absorption ermittelt aus den UVIVIS-Spektren der SmSProben von Tab. 1 gegenüber dem Sputtergraben

Im Folgenden werden die Stöchiometrie, die optischen und elektrischen Eigenschaften der Proben verglichen.

Die Proben 1 und 2 weisen Bereiche mit Stöchiometrie auf und haben nur eine ca. doppelt so hohe Flächenleitfähigkeit wie das Ausgangstarget (Target 1). Leider konnten die Eigenschaften dieser beiden Proben nicht reproduziert werden. Da sich die Beschichtungsrate vom Target 1 nach Ausbau und Lagerung an Luft (für die EDX-Messungen) deutlich verringerte.

Die Beschichtungszeit wurde für Probe 3 so angepasst, dass Probe 1 eine ähnliche Schichtdickenverteilung und eine vergleichbare minimale Flächenleitfähigkeit hat. Probe 4 (DC-Sputtern, angepasste Leistung), sollte wiederum möglichst ähnlich zu Probe 3 (RFSputtern) sein.

Probe 3 hat stöchiometrische Bereiche, die analog Probe 1 eine nur ca. doppelt so hohe Flächenleitfähigkeit hat wie das Ausgangstarget (Target 1). Probe 4 hat im UV/VIS absorptionsarme und im IR transparente Bereiche sowie Bereiche, die eine nur ca. doppelt so hohe Flächenleitfähigkeit haben wie das Ausgangstarget (Target 1). Zusätzlich wurde im IR der Reflexionspeak von SmS nachgewiesen. Leider hat diese Probe an den EDX-Messstellen einen außergewöhnlich hohen Sauerstoffanteil von mehr als $40 \%$.

Probe 5 enthält mehr $S$ als $\mathrm{Sm}$ und ist bis auf den Bereich in der Mitte absorbierend und leitfähig. Auch hier wurde im IR ein Reflexionspeak von SmS nachgewiesen.

Probe 6 (Target 2) enthält mehr $\mathrm{S}$ als $\mathrm{Sm}$ und ist ab ca. $750 \mathrm{~nm}$ absorptionsfrei und isolierend.

Je nach Herstellungsparametern der dünnen SmS-Schichten können gewünschte Schicht- eigenschaften reproduzierbar hergestellt werden.

\section{Schlussfolgerung}

Samarium und Schwefel reagieren mit Sauerstoff und Feuchte. Insofern muss bei Verwendung eines nichtstöchiometrischen Targets nach ersten Sputterversuchen besonders auf die Lagerung der Targets unter Schutzgas oder im Vakuum geachtet werden, um eine Änderung der chemischen Zusammensetzung des Targets zu verhindern. Die gesputterten SmS-Schichten haben Bereiche, bei denen die Eigenschaften auch bei Lagerung an Luft über mehrere Tage stabil bleiben.

Obwohl die SmS-Targets keine stöchiometrische Zusammensetzung hatten, konnten stöchiometrische SmS-Schichten erzeugt werden.

Die SmS-Dünnschichten haben Eigenschaften, die für optische Anwendungen interessant sind. So waren einige SmS-Schichten im VISBereich ab $500 \mathrm{~nm}$ und im IR-Bereich bis $2 \mu \mathrm{m}$ absorptionsarm.

Untersuchungen $\mathrm{zu}$ weiteren Anwendungseigenschaften (z.B. Bestimmung der Dehnungseigenschaften) werden folgen.

\section{Danksagung}

Die Untersuchungen wurden durch Mittel des BMWi ZIM unter den Förderkennzeichen ZF4115801AG5 und ZF4117101AG5 gefördert.

Wir danken Frau Dr. Kunert (TU Dresden) für die thermischen Untersuchungen an den Ausgangsmaterialien, Zwischen- und Endprodukten. Weiterhin danken wir Frau Brünner (TU Dresden) für die REM/EDX-Untersuchungen an den SmS-Pulvern, sowie Frau Kleiner (TU Dresden) und Herrn Dr. Modes (Fraunhofer FEP) für die REM/EDX-Untersuchungen an den SmS-Zwischenprodukten, SmS-Targets und den hergestellten Schichten.

\section{Literatur}

[1] E. Rogers, P.F. Smet, P. Dorenbos, D. Poelman, E. Van Der Kolk, E., Journal of Physics Condensed Matter 22, 1-7 (2010); doi: 10.1088/0953-8984/22/1/015005

[2] N.M. Volodin, Y.N. Mishin, V.V. Kaminskii, Y.Y. Zakharov, Sol Syst Res, 47, 601-604 (2013); doi: 10.1134/S0038094613070150

[3] P. Miodushevsky, M.L. Protopapa, F. de Tomasi, M.R. Perrone, S. Tundo, L. Vasanelli, Thin Solid Films 359 (2000), 251-254 (2000)

[4] Yu. V. Ulashkevich, V.V. Kaminski, A.V. Golubkov, Semiconductors Vol. 43, 305-309 (2009); doi: 10.1134/S1063782609030087 